\title{
Pleuropulmonary Tuberculosis Following Chemotherapy for Lung Cancer at a Tertiary Care Center in India
}

\author{
Navneet Singh, Karan Madan, Ashutosh N Aggarwal, Nalini Gupta, Ashim Das, Digambar Behera
}

\begin{abstract}
Background: Chemotherapy is a risk factor for occurrence of infections. Tuberculosis is a common infection in high prevalence countries. Data on incidence of tuberculosis following lung cancer chemotherapy is limited. The current study was conducted to assess the spectrum and clinical profile of pleuro-pulmonary tuberculosis following lung cancer chemotherapy at a tertiary care institute in North India.
\end{abstract}

Methods: Retrospective data analysis of newly diagnosed lung cancer patients undergoing chemotherapy over a three-andhalf year period. Diagnosis of tuberculosis was made by presence of suggestive clinicoradiological features (fever, cough, pleuritic chest pain, new parenchymal infiltrates or new onset pleural effusion on chest imaging) along with demonstration of acid fast bacilli (AFB) in sputum/pleural fluid/ bronchoalveolar lavage (BAL) fluid and/or growth of Mycobacterium tuberculosis on culture. For biopsy/cytology specimens, tuberculosis was diagnosed by demonstration of granulomatous inflammation with AFB.

Results: Overall six (0.9\%) of 662 lung cancer patients developed tuberculosis [four pulmonary (PTB) and two pleural (TB-PE)]. Occurrence of PTB/TB-PE and of smear-positive PTB after lung cancer chemotherapy was at a rate of 2.59 per 1000 patients/year and 1.73 per 1000 patients/year respectively, compared with national incidences of 1.85 per 1000 population/ year and 0.75 per 1000 population/year respectively. Four patients had objective response to chemotherapy. Diagnosis of PTB/TB-PE was established by sputum microscopy, BAL fluid microscopy and pleural biopsy in two patients each. All patients developed tuberculosis on same side as primary tumor. All patients received standard four-drug anti-tubercular therapy (ATT). Median time interval between diagnosis of lung cancer and that of tuberculosis was 144 (56-317) days while time interval between last chemotherapy cycle and diagnosis of tuberculosis was 55 (36-182) days. Median overall survival and survival after diagnosis of tuberculosis were 312 (141-504) days and 174 (41-199) days respectively.

Conclusion: Occurrence of PTB/TB-PE after lung cancer chemotherapy is higher than the corresponding incidence of tuberculosis observed in the general Indian population. Overall survival of these patients is similar to lung cancer patients in general if clinical suspicion of tuberculosis is actively pursued and ATT initiated promptly following diagnosis of tuberculosis.

Keywords: Chemotherapy, Lung cancer, Pleural, Pulmonary, Tuberculosis.

How to cite this article: Singh N, Madan K, Aggarwal AN, Gupta N, Das A, Behera D. Pleuropulmonary Tuberculosis Following Chemotherapy for Lung Cancer at a Tertiary Care Center in India. J Postgrad Med Edu Res 2013;47(4):177-180.

Source of support: Nil

Conflict of interest: None declared

\section{INTRODUCTION}

Chemotherapy administration is a risk factor for occurrence of infections. Tuberculosis is a common infection among people residing in endemic (high prevalence) countries. Both reactivation of latent foci of old tuberculous infection as well as reinfection with a new strain of Mycobacterium tuberculosis (MTB) contribute in general to the high incidence of tuberculosis disease in endemic countries. A number of mechanisms may lead to a higher incidence of tuberculosis in patients with cancer including those with lung cancer. These include impairment of immune responses due to malignancy per se, malnutrition or secondary immunosuppression due to the effects of cytotoxic chemotherapy. In the context of lung cancer, pulmonary tuberculosis can precede, occur concurrently with or subsequent to the diagnosis of lung cancer. ${ }^{1-4}$ The diagnosis of pulmonary tuberculosis in patients with lung cancer can be delayed due to the preexisting radiological abnormalities associated with the latter. Previous reports have pooled descriptions of lung cancer patients in whom active pulmonary tuberculosis and lung cancer occurred concurrently with those in whom pulmonary tuberculosis followed management of lung cancer. ${ }^{2}$ The influence of occurrence of PTB on outcomes of lung cancer patients is also unclear. ${ }^{1}$ We have assessed the occurrence of pleuropulmonary tuberculosis following chemotherapy for lung cancer at our tertiary care institute in North India and provide a description of their demographic and treatment-related characteristics as well as outcomes in this paper.

\section{METHODS}

This was a retrospective analysis of routinely collected data on lung cancer patients presenting to the authors' institute. As per the normal protocol of our clinic, diagnosis of lung cancer is confirmed by cytological/histopathological examination of tumor specimens obtained from accessible sites in all patients. Coexistence of active tuberculosis is also ruled out simultaneously by microscopic examination for presence of acid fast bacilli (AFB) in sputum or other representative specimens obtained during the process of diagnostic confirmation of lung cancer.

Consecutive newly diagnosed patients, with cytologically/ histopathologically proven lung cancer, who were initiated on chemotherapy at the lung cancer clinic of the authors' institute over a three-and-half year period (January 2008- 
June 2011) comprised the study population. Demographic characteristics (including age, gender and performance status), histological type, stage of disease and details of smoking status were noted for all patients and have been described in detail previously. ${ }^{5-7}$ Chemotherapy regimens and normal management protocols used at our center have been described in detail elsewhere. ${ }^{8,9}$ Case records of the study population described above were retrieved and patients in whom the diagnosis of pleuro-pulmonary tuberculosis was established subsequent to initiation of chemotherapy were identified and selected for the purpose of the current analysis. For the sake of uniformity in relation to stage description for this analysis, we used the 7th edition of TNM staging. ${ }^{10}$ Criteria used for establishing diagnosis of tuberculosis included presence of suggestive clinicoradiological features (fever, cough, pleuritic chest pain, new parenchymal infiltrates or new onset pleural effusion on chest imaging) along with demonstration of AFB on smears prepared from sputum, pleural fluid or other representative specimens and/ or growth of MTB on their culture. In case of biopsy/ cytology specimens, demonstration of granulomatous inflammation with AFB was taken as diagnostic for tuberculosis. Clinical profile and outcomes of patients with pleuropulmonary tuberculosis following administration of chemotherapy are presented herein in a descriptive fashion [percentages, mean, (standard deviation) or median (interquartile range)]. All analyses were performed using the statistical software SPSS version 15.0 (SPSS Inc, Chicago, IL, USA).

\section{RESULTS}

Of 763 patients diagnosed during the study period, those excluded from analysis were 14 who did not receive any form of definitive treatment, 23 with nonbronchogenic histologies and 64 who received oral epidermal growth factor receptor tyrosine kinase inhibitors as first line (sole) treatment modality for NSCLC. The remaining 662 lung cancer patients who received chemotherapy comprised the study population. Overall, six $(0.9 \%)$ patients were diagnosed with tuberculosis (four pulmonary in addition to the two with pleural). The diagnosis was established by sputum microscopy, bronchioalveolar lavage fluid microscopy and pleural biopsy in two patients each. Four of the six patients had an objective response to chemotherapy as per RECIST criteria. ${ }^{11}$ Tubercular involvement of right lung/pleura occurred in five patients. All patients developed tuberculosis on the same side as the primary tumor. All patients were treated with standard four-drug ATT. Individual case descriptions are provided in Table 1 while pooled demographic characteristics as well as treatment and survival profile of these patients is presented in Table 2. In the current study, occurrence of pleuropulmonary tuberculosis and of smear-positive pulmonary tuberculosis among lung cancer patients receiving chemotherapy was at a rate of 2.59 per 1000 patients/year and 1.73 per 1000 patients/year respectively, compared with national incidences of 1.85 per 1000 population/year and 0.75 per 1000 population/year respectively. ${ }^{12,13}$

\section{DISCUSSION}

Approximately, one-third of the global population is infected with the MTB bacillus and it is estimated that, in India, the prevalence of infection is $40 \% .^{12}$ The global incidence of tuberculosis disease in 2010 was estimated at 128 per $1,00,000$ population [ $95 \%$ confidence interval (CI) of 123-133] with that in India being 185 per 1,00,000 population (95\% CI of 167-205). ${ }^{13}$ Of these, new smear positive pulmonary tuberculosis cases in India are estimated to be

\begin{tabular}{|c|c|c|c|c|c|c|c|}
\hline S. no. & $\begin{array}{l}\text { Age/ } \\
\text { gender }\end{array}$ & $\begin{array}{l}\text { Histology smoking } \\
\text { status }\end{array}$ & $\begin{array}{l}\text { TNM status } \\
\text { stage }\end{array}$ & $\begin{array}{l}\text { Cycles regimen } \\
\text { response }\end{array}$ & $\begin{array}{l}\text { Site of } 1^{\circ} \text { tumor } \\
\text { Site of TB lesion }\end{array}$ & $\begin{array}{l}\text { Type of TB } \\
\text { Mode of } D x\end{array}$ & $\begin{array}{l}\text { OS } \\
\text { ival after TB Dx }\end{array}$ \\
\hline \multirow[t]{2}{*}{1.} & $21 / F$ & SCLC & T4N2M1b & $6 ; \mathrm{IC}$ & LUL & Pulmonary & 499 \\
\hline & & NS & Stage IV (ED) & PR & LUL & BAL microscopy & 196 \\
\hline \multirow[t]{2}{*}{2.} & 49/M & SCLC & T2aN2M1b & $6 ; \mathrm{IC}$ & RUL & Pleural & 363 \\
\hline & & NS & Stage IV (ED) & PR & Rt. pleura & Closed pleural biopsy & 189 \\
\hline \multirow[t]{2}{*}{3.} & $53 / M$ & NSCLC (SqCC) & T3N2M0 & 2; DC & RLL & Pulmonary & 260 \\
\hline & & $\mathrm{Sm}$ & Stage IIIA & SD & RUL & Sputum microscopy & 206 \\
\hline \multirow[t]{2}{*}{4.} & $63 / M$ & NSCLC (ADC-SqCC) & T3N2M0 & $5 ; P C$ & RLL & Pulmonary & 518 \\
\hline & & Sm & Stage IIIA & PR & RUL & Sputum microscopy & 159 \\
\hline \multirow[t]{2}{*}{5.} & $38 / \mathrm{M}$ & NSCLC (ADC) & T2aN2M1b & 4; PemC & RLL & Pleural & 166 \\
\hline & & NS & Stage IV & PR & Rt. pleura & $\begin{array}{l}\text { Thoracoscopic } \\
\text { pleural biopsy }\end{array}$ & 52 \\
\hline \multirow[t]{2}{*}{6.} & $60 / \mathrm{M}$ & NSCLC (SqCC) & T4N3M0 & $2 ; \mathrm{DC}$ & RLL & Pulmonary & 64 \\
\hline & & $\mathrm{Sm}$ & Stage IIIB & $\mathrm{SD}$ & RUL & BAL microscopy & 8 \\
\hline
\end{tabular}

M: Male; F: Female; T: Tumor; N: Nodal; M: Metastasis; Dx: Diagnosis; OS: Overall survival; ED: Extensive disease; BAL: Bronchoalveolar lavage; 1: Primary; TB: Tuberculosis; LUL: Left upper lobe; RUL: Right upper lobe; RLL: Right lower lobe; Rt. Right; SM: Current/ex smoker; NS: Never-smoker; SCLC: Small cell lung cancer; SqCC: Squamous cell carcinoma; ADC: Adenocarcinoma; DC: Docetaxelcisplatin; PC: Paclitaxel-cisplatin; IC: Irinotecan-cisplatin; PemC: Pemetrexed-cisplatin; PR: Partial response; SD: Stable disease 


\begin{tabular}{ll}
\hline \multicolumn{2}{c}{ Table 2: Pooled characteristics of six patients with pleuropulmonary tuberculosis } \\
\hline Age $^{*}$ & $47.3(15.6)$ years \\
Males & $5(83.3 \%)$ \\
Current/ex-smokers & $3(50.0 \%)$ \\
Histology & $2(33.3 \%)$ \\
- Squamous $\quad$ Small cell & $2(33.3 \%)$ \\
- Non-squamous NSCLC & $2(33.3 \%)$ \\
Stage IIIB-IV at lung cancer diagnosis & $4(66.7 \%)$ \\
Extrathoracic metastasis at lung cancer diagnosis & $3(50.0 \%)$ \\
Baseline performance status of ECOG 0-1 & $5(83.3 \%)$ \\
Cycles of chemotherapy & $4.5(2-6)$ \\
Objective response to chemotherapy & $4(66.7 \%)$ \\
- Partial response & $2(33.3 \%)$ \\
Time interval between diagnosis of lung cancer and diagnosis of tuberculosis & $144(56-317)$ days \\
Time interval between last chemotherapy cycle and diagnosis of tuberculosis & $55(36-182)$ days \\
Overall survival & $312(141-504)$ days \\
Survival after diagnosis of tuberculosis & $174(41-199)$ days \\
\hline
\end{tabular}

Values expressed as percentages and as median (interquartile range) unless specified otherwise; *Mean (standard deviation); NSCLC: Non-small cell lung cancer

75 per 100,000 population per year. ${ }^{12} \mathrm{~A}$ number of malignancies including lung cancer have been associated with the occurrence of tuberculosis. ${ }^{2,14-16}$ Previous reports indicate that among patients with lung cancer who also have a diagnosis of active tuberculosis, the diagnosis of tuberculosis is made in approximately $70 \%$ of these subjects subsequent to the diagnosis of lung cancer. ${ }^{2,17}$

Overall, occurrence of tuberculosis following anticancer chemotherapy has been an infrequently described clinical scenario. Although majority of the global burden of tuberculosis is concentrated in the developing world, these regions of the world often do not have adequate facilities for diagnosis, treatment and follow-up of cancer patients for the majority of the population residing therein. Data from the current study suggest that both pleuropulmonary tuberculosis and smear positive pulmonary tuberculosis were more frequent than in the general Indian population.

Published data on pulmonary tuberculosis among lung cancer patients is sparse. A retrospective case control study described 24 patients with tuberculosis following anticancer chemotherapy for a broad group of primary malignancies. ${ }^{16}$ Of these, only two patients had underlying lung cancer. Pulmonary tuberculosis was the commonest form of involvement in both cases and controls $(87.5 \%$ and $79.2 \%$ respectively). Cavitation and multilobar involvement was similar in both groups. Another series reported 36 lung cancer patients who were diagnosed with tuberculosis either concurrently or sequentially. ${ }^{2}$ Of these, 26 (72.2\%) were diagnosed with tuberculosis subsequent to the diagnosis of lung cancer with a median time to diagnosis of 4 months (range 1 to 47 months). Chemotherapy with/without other treatment modalities had been administered to $22(61.1 \%)$ patients who developed tuberculosis. Squamous cell carcinoma $(61.1 \%)$ was the most common histological type. The median time to survival of lung cancer patients from the time of diagnosis of tuberculosis was 17.3 months. Progression of lung cancer was the most common cause of death in the lung cancer group and none of the deaths were attributable to tuberculosis per se. The authors had suggested that respiratory tuberculosis, if treated adequately, does not influence the clinical course of lung cancer subjects.

In our study also, the median overall survival for lung cancer patients who developed pleuropulmonary tuberculosis was 312 days (approximately 10.5 months) which is similar to that observed for patients with advanced lung cancer in general at our centre. ${ }^{8}$ However, the fact remains that pulmonary tuberculosis occurring in the setting of lung cancer represents a clinical scenario that is often difficult to diagnose. It is therefore important for physicians managing lung cancer patients in countries with high prevalence of tuberculosis to make all efforts at getting an early confirmation of the diagnosis once it has been suspected since as well as to initiate timely and appropriate ATT. It has been suggested that detection of a tree-in-bud pattern on high resolution CT scan of lung parenchyma may be an indicator of active pulmonary tuberculosis in the presence of suggestive symptoms and should prompt an aggressive workup for confirming diagnosis of active tuberculosis in these patients. ${ }^{2}$

Occurrence of lung cancer following pulmonary tuberculosis and vice versa as well as coexistence of pulmonary tuberculosis with lung cancer all have been described. ${ }^{1-4}$ However, individual descriptions of pleural tuberculosis following chemotherapy for lung cancer have not been previously published. The two cases of pleural tuberculosis which were included in the analysis of the 
current study, to the best of the authors' knowledge, represent the first two cases of pathologically proven pleural tuberculosis following chemotherapy for lung cancer and their details have been reported separately. ${ }^{18}$

\section{SUMMARY}

Our review of the records of more than 650 patients undergoing chemotherapy for lung cancer indicates that although occurence of pleuropulmonary tuberculosis in this high-risk patient population is higher than that observed in the general Indian population, tuberculosis does not seem to adversely influence overall survival if an aggressive policy of actively pursuing the clinical suspicion of tuberculosis is adopted and ATT promptly initiated once diagnosis has been established by microscopic or pathological means.

\section{REFERENCES}

1. Chen YM, Chao JY, Tsai CM, Lee PY, Perng RP. Shortened survival of lung cancer patients initially presenting with pulmonary tuberculosis. Jpn J Clin Oncol 1996;26:322-327.

2. Cha SI, Shin KM, Lee JW, Lee SY, Kim CH, Park JY, Jung TH. The clinical course of respiratory tuberculosis in lung cancer patients. Int J Tuberc Lung Dis 2009;13:1002-1007.

3. Cicenas S, Vencevicius V. Lung cancer in patients with tuberculosis. World J Surg Oncol 2007;5:22.

4. Liang HY, Li XL, Yu XS, Guan P, Yin ZH, He QC, Zhou BS. Facts and fiction of the relationship between preexisting tuberculosis and lung cancer risk: a systematic review. Int J Cancer 2009;125:2936-2944.

5. Singh N, Aggarwal AN, Gupta D, Behera D, Jindal SK. Unchanging clinico-epidemiological profile of lung cancer in North India over three decades. Cancer Epidemiol 2010;34:101-104.

6. Singh N, Aggarwal AN, Gupta D, Behera D. Prevalence of low body mass index among newly diagnosed lung cancer patients in North India and its association with smoking status. Thoracic Cancer 2011;2:27-31.

7. Singh N, Aggarwal AN, Gupta D, Behera D, Jindal SK. Quantified smoking status and non-small cell lung cancer stage at presentation: analysis of a North Indian cohort and a systematic review of literature. J Thorac Dis 2012;4:474-484.

8. Singh N, Aggarwal AN, Behera D, Jindal SK. Intercycle delays during chemotherapy of non-small cell lung cancer in a health care resource-constrained setting and their effect on overall survival. J Thorac Oncol 2010;5:236-239.

9. Singh N, Aggarwal AN, Behera D. Management of advanced lung cancer in resource-constrained settings: a perspective from India. Expert Rev Anticancer Ther 2012;12:1479-1495.

10. International Association for the Study of Lung Cancer. Staging Manual in Thoracic Oncology, 1st ed. Orange Park, FL, USA: Editorial Rx Press, 2009.

11. Therasse P, Arbuck SG, Eisenhauer EA, Wanders J, Kaplan RS, Rubinstein L, Verweij J, Van Glabbeke M, van Oosterom AT, Christian MC, et al. New guidelines to evaluate the response to treatment in solid tumors. European Organization for Research and Treatment of Cancer, National Cancer Institute of the United States, National Cancer Institute of Canada. J Natl Cancer Inst 2000;92:205-216.

12. TB India 2011: Revised National Tuberculosis Control Programme Annual Status Report, vol. 2012, New Delhi: Central TB Division, Directorate General of Health Services, Ministry of Health and Family Welfare, Government of India, 2011. Available at: http://www.tbcindia.nic.in/pdfs/RNTCP\% 20TB\%20India\%202011.pdf.

13. Global Tuberculosis Control 2011, vol. 2012, Geneva: World Health Organization, 2011. Available at http://www.who. int/tb/ publications/global_report/en/.

14. Kaplan MH, Armstrong D, Rosen P. Tuberculosis complicating neoplastic disease. A review of 201 cases. Cancer 1974;33: 850-858.

15. Karnak D, Kayacan O, Beder S. Reactivation of pulmonary tuberculosis in malignancy. Tumori 2002;88:251-254.

16. Kim DK, Lee SW, Yoo CG, Kim YW, Han SK, Shim YS, Yim JJ. Clinical characteristics and treatment responses of tuberculosis in patients with malignancy receiving anticancer chemotherapy. Chest 2005;128:2218-2222.

17. Libshitz HI, Pannu HK, Elting LS, Cooksley CD. Tuberculosis in cancer patients: an update. J Thorac Imaging 1997;12:41-46.

18. Madan K, Singh N, Das A, Behera D. Pleural tuberculosis following lung cancer chemotherapy: a report of two cases proven pathologically by pleural biopsy. BMJ Case Rep 2013; doi:10.1136/bcr-2012-008196:Published 22 January 2013.

\section{ABOUT THE AUTHORS}

\section{Navneet Singh (Corresponding Author)}

Assistant Professor, Department of Pulmonary Medicine, Postgraduate Institute of Medical Education and Research, Chandigarh, India e-mail: singh.navneet@pgimer.edu.in

\section{Karan Madan}

Senior Resident, Department of Pulmonary Medicine, Postgraduate Institute of Medical Education and Research, Chandigarh, India

\section{Ashutosh N Aggarwal}

Additional Professor, Department of Pulmonary Medicine, Postgraduate Institute of Medical Education and Research, Chandigarh, India

\section{Nalini Gupta}

Associate Professor, Department of Cytology and Gynecological Pathology, Postgraduate Institute of Medical Education and Research, Chandigarh, India

\section{Ashim Das}

Professor, Department of Histopathology, Postgraduate Institute of Medical Education and Research, Chandigarh, India

\section{Digambar Behera}

Professor, Department of Pulmonary Medicine, Postgraduate Institute of Medical Education and Research, Chandigarh, India 